\section{Imaging of Big and Messy Biological Structures Using Electron Tomography}

\author{
Gina E. Sosinsky and Maryann E. Martone \\ University of California
}

Any student of cell biology cannot help but be impressed with the exquisite organization of cellular and subcellular compartments, where individual proteins and even nucleic acids are targeted to specific domains and held together in macromolecular assemblies to achieve orderly and efficient interactions. Perhaps nowhere is the specialization of cellular compartments more apparent than in the nervous system, where the cell contains multiple, well-defined functional domains, each with its own unique biochemical character. The nervous system is also unique in the large number of gene products expressed and their heterogeneous distribution, not only within cells but also between different cell populations. Over half of the genes identified in the human genome are thought to be expressed in the nervous system. To realize the full impact of the genomic and proteomic efforts of the last decade, structural complexity in biological systems must be addressed at scales of resolution ranging from $1 \mathrm{~nm}$ to $100 \mu \mathrm{m}$ and up. New tools are needed to tackle one of the next grand challenges in biological science: situating proteins and macromolecular complexes within their cellular and tissue-specific environments.

Technological improvements in both light and electron microscopic imaging have led to major advances in our ability to fill in missing information between the molecular and cellular realms. At the light microscopic level, computational image restoration techniques and the optical sectioning capabilities of the confocal and multiphoton microscopes are increasing our ability to retrieve $3 D$ and $4 D(3 D+$ time) information at the resolution achievable with optical-based imaging techniques. At the electron microscopic level, electron tomography is revealing new information about the 3D ultrastructure of tissues, cells and macromolecular complexes (as reviewed in references 1-3). Using tomography, a 3D structure (also referred to as a tomogram) is obtained containing all internal substructures with the potential of mapping the location and segregation of functionally important macromolecules in $3 \mathrm{D}$ at much higher resolution than can be achieved by serial sectioning (4-7). Electron tomography is filling in large gaps in our knowledge about structures in the $5-40 \mathrm{~nm}$ range because of the ability to generate computed slices through a 3D volume at much higher resolution than can be achieved by physical sectioning. It also has proven useful in combination with high voltage electron microscopy for the study of overall cell and tissue organization on a large scale, bridging the gap between light and electron microscopy.

Electron tomography is moving from a specialized experimental technique practiced by a few laboratories to one that is delivering critical new information to cell biologists, structural biologists and neuroscientists. This migration has been fueled in part by increased computational power and the availability of higher voltage electron microscopes with computer controlled specimen stages, column optics and digital image recording. Electron tomography has led to major revisions in our understanding of even well studied structures such as the mitochondria and the Golgi apparatus. In fact, electron tomography of frozen specimens ("cryotomography") was named a runner-up "breakthrough of the year" by Science magazine for 2002 (8). Three of the National Institutes of Health Research Resources: The Boulder Laboratory for 3-Dimensional Electron Microscopy of Cells at the University of Colorado (Boulder, Colorado), The Resource for the Visualization of Biological Complexity at the Wadsworth Center (Albany, New York) and The National Center of Microscopy and Imaging Research at the University of California, San Diego (La Jolla, California) are dedicated to the application and further advancement of electron tomographic imaging and reconstruction technology (9).

Tomography is a generalized method for reconstructing the interior of an object in $3 \mathrm{D}$ from independently acquired $2 \mathrm{D}$ views or projections. Examples of tomography applications include not only those for electron microscopy (called electron tomography), but also computerized axial tomography (CAT)-scan imaging for medical imaging. The principles of the data collection and reconstruction are the same in spite of the different resolution scales and specimen preparation methods. The Austrian mathematician Johann Radon derived the mathematical formulation for tomographic reconstruction in 1917. While the theory behind tomographic reconstruction was proposed over 85 years ago, implementation of tomographic imaging technology started with the age of modern computing in the 1970's. Two Nobel prizes were awarded that directly related to $3-D$ reconstructions methods used in tomographic reconstruction: one shared by Allan M. Cormack and Godfrey N. Hounsfield in 1979 for the development of CAT-scanning and one, in 1982, to Aaron Klug, in part for his development of 3D reconstruction methodology of molecular structures using electron microscopy.

Tomography involves recording images over a broad angular range so that the $3 \mathrm{D}$ structure will have adequate resolution along the three axes. For a volume determined by electron tomography, the three axes, $X, Y$ and $Z$, are defined by the optical setup of the electron microscope. The $Z$ axis is usually defined as the one parallel to the electron beam, whereas $X$ and $Y$ are perpendicular to the optical axis. For tomography, the $Y$ axis usually refers to the tilt axis. For the type of tomography discussed here, the specimen typically consists of a section of biological material prepared for electron microscopy. Tomography consists of four major steps: data acquisition, image alignments, reconstruction and visualization and analysis of the reconstruction (see Fig. 1). In tomographic techniques, either the specimen or detector is rotated to obtain each projection. In the electron microscope, it is the specimen that is tilted along a single axis from $-60^{\circ}$ to $60^{\circ}$ with images (projections) recorded at equal 1 to $2^{\circ}$ increments. Many factors affect the resolution achievable in a given reconstruction. One of the main determinants is the angular range over which the specimen is sampled. Tilt images generally cannot be recorded over a full $360^{\circ}$ due to physical limitations imposed by specimen holders, specimen grids and tilt stages. The limited angular range leads to a "missing wedge" of information, resulting in decreased resolution in the $Z$ axis compared to the $X$ and $Y$ (c.f. reference(10). Finer increments between consecutive tilts and acquiring data at higher tilt angles $\left(\geq \pm 60^{\circ}\right.$ ) usually result in an Increase in the $Z$ axis resolution. Conversely, for a fixed tilt range and increment, the resolution decreases as the thickness of the section increases (11). For resolving molecular structures in complex cellular and tissue contexts, section thickness is typically limited to $0.5 \mu \mathrm{m}$ for microscopes of $400 \mathrm{keV}$. To increase the extent of reconstruction, a method called serial section electron tomography is employed, whereby serial thick sections are be obtained and reconstructed. The resulting volumes in the series are aligned and merged into a single volume $(12-14)$. When molecular resolution is not required, as in $3 \mathrm{D}$ studies of higher order complex cellular structures within a tissue, sections up to $4 \mu \mathrm{m}$ have been employed with success in combination with ultra high voltage electron microscopy ( $3 \mathrm{MeV},(15 ; 16)$. These thicker sections have been used to measure dendritic spines in neuronal spiny dendrites (as seen in Figure 2C-E). In this case, the dendrites are 100 's of microns in length, while the spines have features of interest $-50 \mathrm{~nm}$ in diameter. The UHVEM is necessary to image such thick sections because the thickness of the section increases as the inverse of the cosine of the tilt angle so that at $60^{\circ}$ and at $70^{\circ}$ tilt, the amount of specimen that the electron must travel through is two and three times, respectively, that at $0^{\circ}$ and that one should use an electron microscope with appropriate penetration power.

Prior to data acquisition, some specimen dependent choices must be made for determining imaging and computational parameters that, in the end, will provide the best reconstruction containing clearly detectable features. For some specimens whose features follow a long central axis, such as a nerve or macrofiber, it is advantageous to orient the area of interest along the tilt axis, because the resolution along the tilt axis will always be optimal compared to the axis perpendicular to the tilt axis. To equalize the resolution between the $X$ and $Y$ axis, double tilt tomography has been employed which involves the merging of data using two orthogonal single axis tilt series. Because this procedure decreases the missing wedge of information, resolution is also 


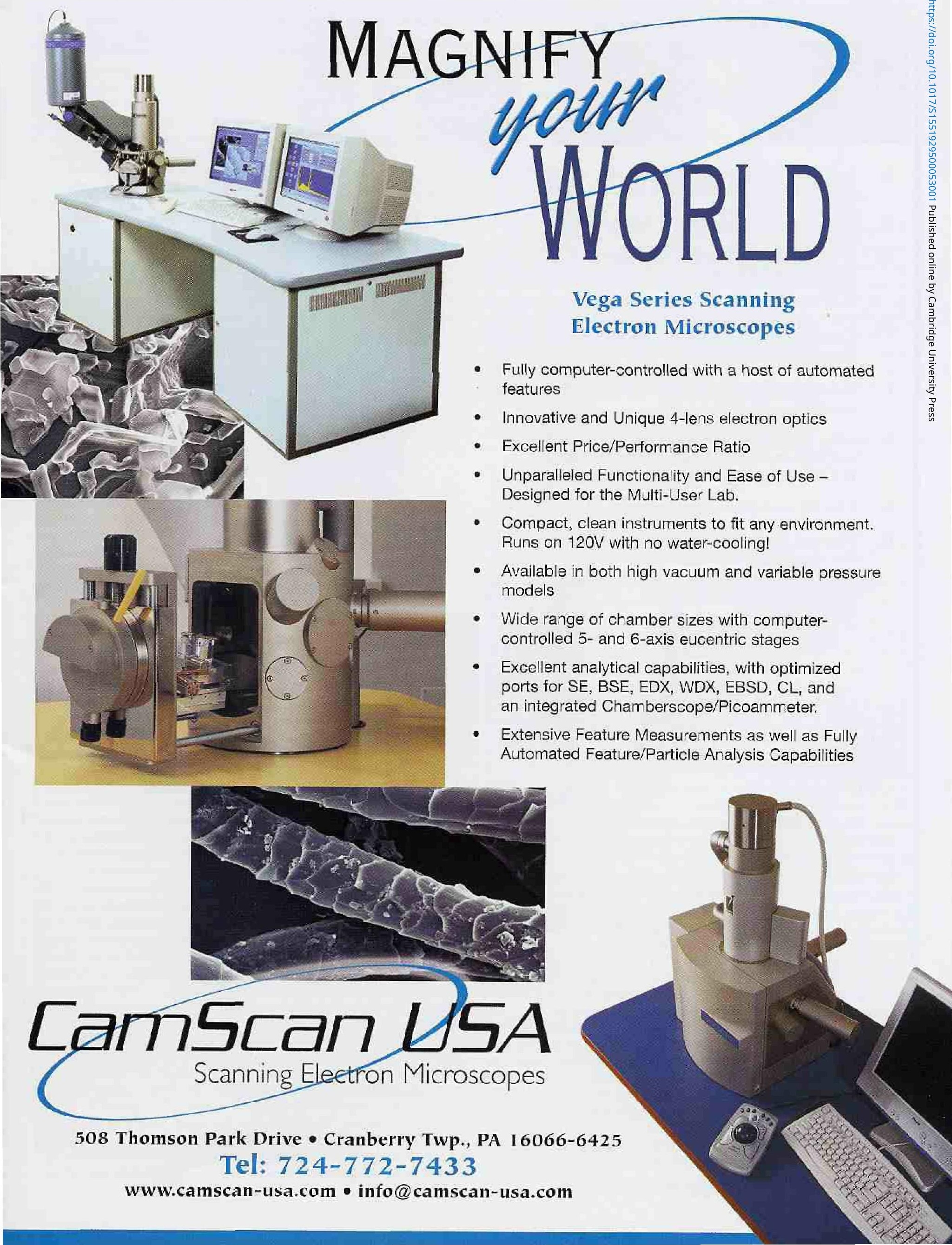



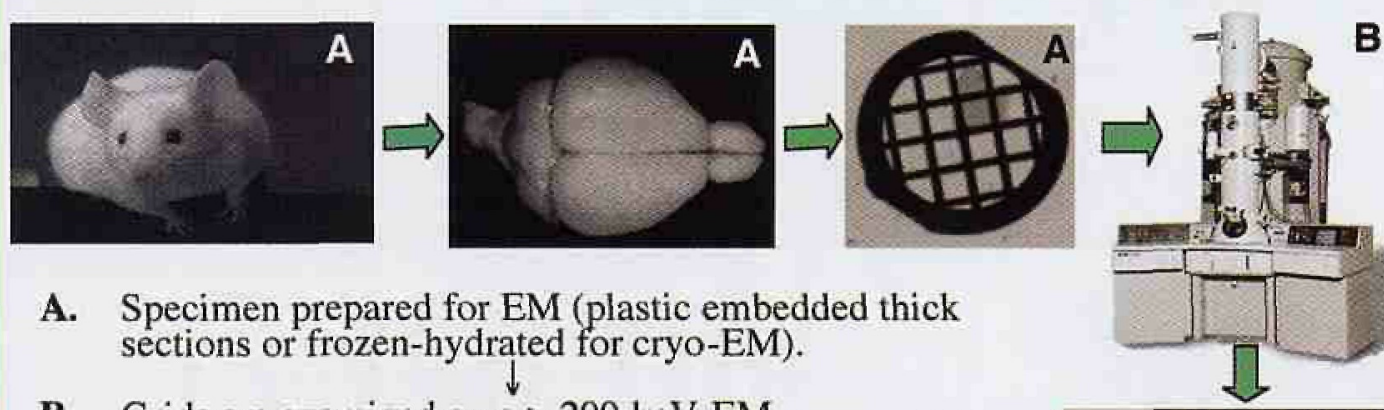

A. Specimen prepared for EM (plastic embedded thick sections or frozen-hydrated for cryo-EM).

B. Grids are examined on $\mathrm{a} \geq 200 \mathrm{keV}$ EM.

C. A tilt series is collected, typically with $1-2$ increments between images. Images digitized if on film.

D. Tilt images are aligned to a common axis by calculating positions of commonfiducial marks or correlational alignments.

E. Rotational and translational shifts applied to all images.

F. After normalization and corrections for tilt and warpage, a volume is calculated using back projection or re-iterative algorithms.

G. The $3 \mathrm{D}$ reconstruction is viewed.

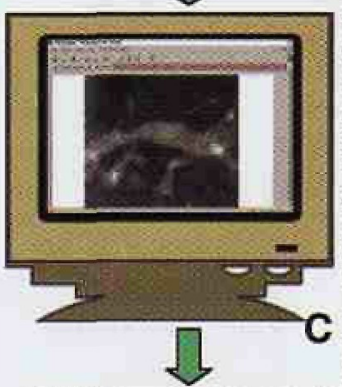

methods of aligning the images to each other are commonly used. The first is a correlational approach that calculates the rotation and translation vectors based on cross- and auto-correlation functions, however, for this approach to work the data in the individual tilts should not vary much between consecutive tilt images and is therefore, used in conjunction with cosine weighted angle data acquisition and with thin samples (21). The second approach is to use fiducial marks whereby gold beads or other electron dense objects are used to align the image data to a common origin. Colloidal gold is commonly applied to the top and/or bottom of the section or layered onto an aqueous sample. as is the practice for tomographic imaging of isolated macromolecular structures. Computer programs track the fiducials and calculate a least squares fit of the fiducial markers. Depending on the specific least squares fitting alignment algorithm used, the minimum number of gold beads necessary to track a series is $-6-8$ for each image and these markers need to be common to elther the entire stack of images or a sizable subset of consecutive images. One of the benefits of using the fiducial marker alignment approach over correlational alignments is that

F, G these fiducials can be used as an independent measure of specimen or image distortions and "out of plane" tilt of the sample. The rotation and translation vectors determined from this fitting procedure are then applied to each of images. In general, the greater the number of fiducials, the more precise the alignment of the image data set and the better the quality of reconstruction. The latter can be seen in the clarity and distinctiveness of the features such as membranes, microtubules or neurofilaments within the volume.

steps in electron tomography (see text for details).

improved along the $Z$ axis $(17 ; 18)$. Considerations for determining a magnification for data acquisition are based on either defining an "area of interest" or determining a resolution necessary for imaging specific features within the structure. In the first criterion, the magnification is determined so that the image will contain the entire area of interest. In the latter criterion, in order to determine if an image or tomogram will deliver sufficient resolution to image specific features, the final pixel size must be calculated based on pixel size of the recording medium divided by the microscope magnification. If images are collected on film, these are digitized at a sufficiently fine scan raster. For both film and CCD images, the magnification must be determined so that the pixel sampling on the specimen obeys the Shannon sampling criterion for resolving features (19). However, an additional criterion for determining magnification and number of images collected may be the radiation sensitivity of the sample. Radiation sensitivity is particularly important with unstained, native specimens (20).

The quality of the tomographic reconstruction is absolutely dependent upon the precision with which the tilt series can be aligned. Two 


\section{LEO SUPRA...}

\section{The ultra-high resolution, variable pressure analytical FESEM}

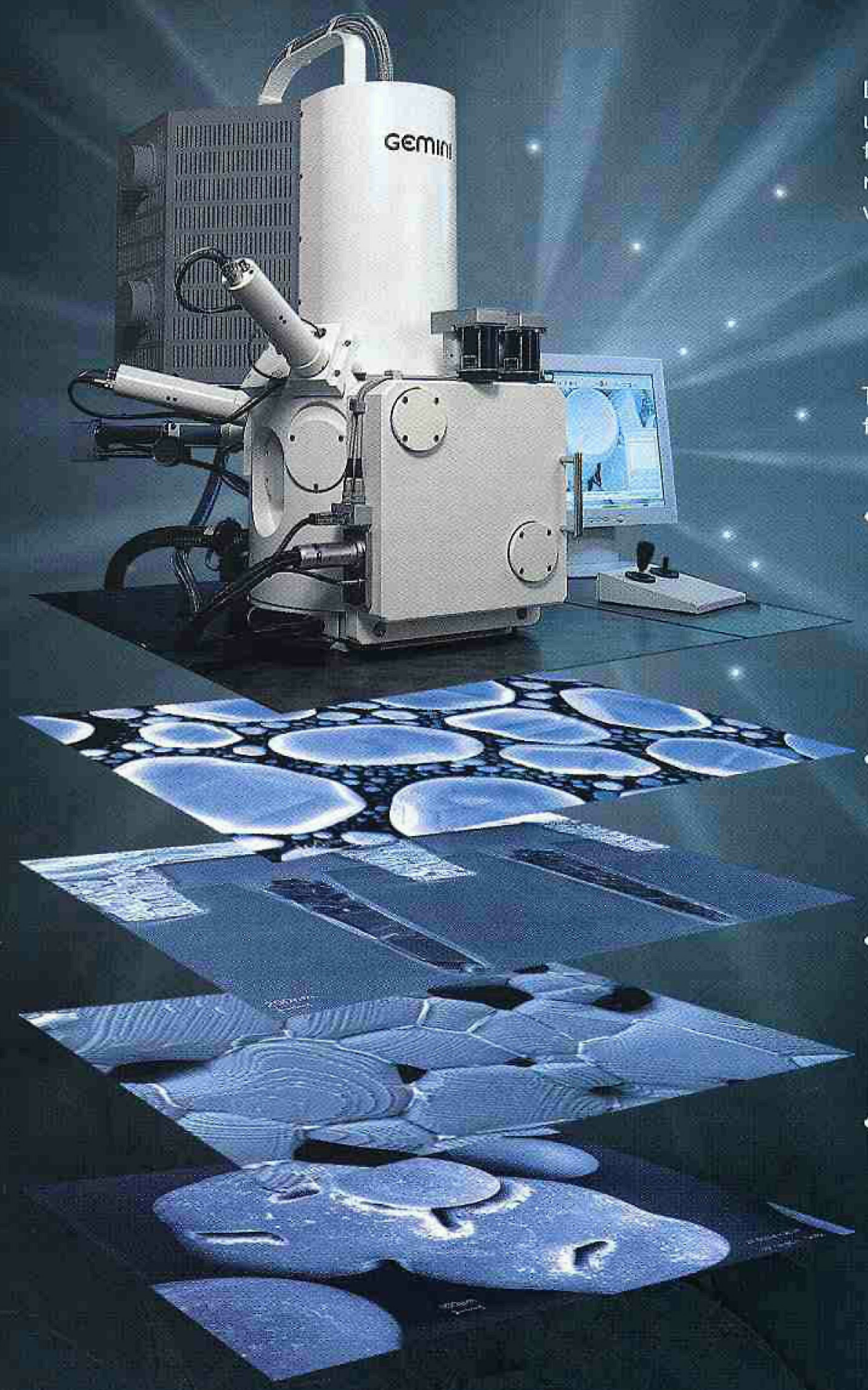

LEO SUPRA is the versatile, ultra-high resolution FESEM for solutions to semiconductor, materials, life science and variable pressure applications.

The new LEO SUPRA combines four instruments into one:

* The LEO SUPRA delivers ultra-high resolution over the complete voltage range: $1.0 \mathrm{~nm} @ 15 \mathrm{kV}$

$1.7 \mathrm{~nm} @ 1 \mathrm{kV}$

$4.0 \mathrm{~nm} @ 0.1 \mathrm{kV}$

- Large sample chamber and fully motorized precision stage handles oversized specimens

- Full analytical FESEM with probe currents up to $20 \mathrm{nA}$ for EBSD and WDX applications

- LEO's proprietary variable pressure technology allows investigation of non-conducting specimens by eliminating charging without prior sample preparation

LEO Electron Microscopy Group A Carl Zeiss SMT AG Company 73417 Oberkochen, Germany Tel: ++497364946137 Fax: $++497364-944851$ info@leo,de • wwwleo-em.com
LEO Electron Microscopy Inc A Carl Zeiss SMI AG Company Thornwood, New York 1059 A Toll Free: $800-356-1090$ Fax: $914-681-7443$ wwwleo usa.com 
tion algorithms using density values within the tomogram. Segmentation is often the most time consuming part of the process because of the human intervention and a priori knowledge required to interpret the volumes.

Contrast is critical in determining the visibility and reliability of features within a tomogram. In plastic embedded biological material, contrast is mainly due to deposition of osmium, uranyl acetate and lead. For macromolecular complexes in suspension, the source of contrast can either arise from negative stain or, in vitrified samples, by adjusting the defocus settings to maximize density differences between protein, ice, lipid and nucleic acids.

Several groups have been pushing the resolution of tomographic reconstructions to reveal the structure of molecular specializations in situ and fitting these structures with higher resolution structures derived from cryomicroscopy and $\mathrm{X}$-ray crystallography (5). Most of these studies employ imaging of unfixed and unstained preparations using cryo-tomography. While there have been some landmark achievements in cryo-electron tomography such as the recent work from Baumeister's laboratory (6). interoretable reconstructions have been limited to either thin areas of cells, isolated complexes or organelles (25-27) or gutted cells (5). This limitation is mainly due to the interpretability of overlapping structures found in the dense cell cytoplasm. The goal in each of these was the recognition of well-studied cellular components such as mitochondria, chaperones, proteosomes or actin filaments. Our sister Research Resources at Albany, and Boulder have had active roles in developing and extending this methodology in cellular imaging of unstained and vitreous specimens as well as specimens conventionally prepared.

At NCMIR, we seek to understand how proteins map onto complex cellular microdomains like synapses, and how these domains in turn relate to higher orders of structure (see examples of such large scale structures in Fig. 2). Although cryo-tomography of unstained material offers the opportunity to study close to native molecular structure in situ (28), this approach is not often feasible for many interesting questions in cell biology, particularly in the nervous system. The brain is the most highly differentiated and helerogeneous of all tissues. Each cubic micron of neuropil can contain dozens of cellular components, each with their own unique macromolecular specializations, and these components vary from brain region to brain region. Determining the molecular specializations of these domains requires the use of selective stains to highlight proteins of interest. Additional information is often required from light microscopic imaging of the same tissue both to locate sparsely distributed proteins or to provide clues to the identity of the cell where they are located. In addition, the revolution in light
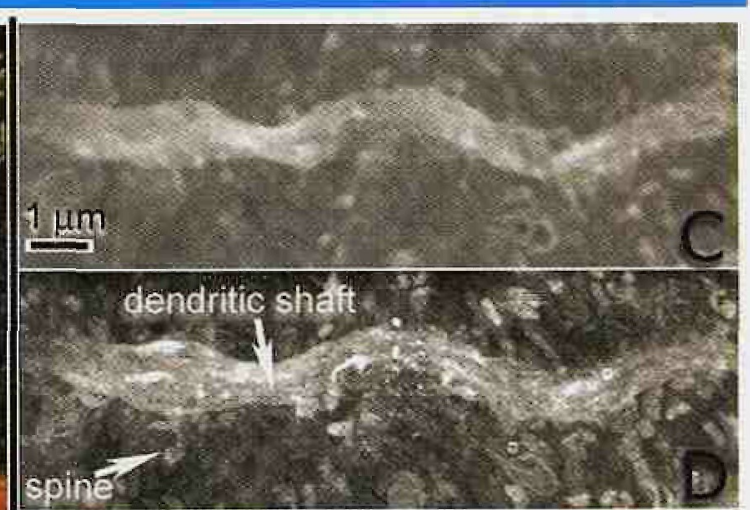

A
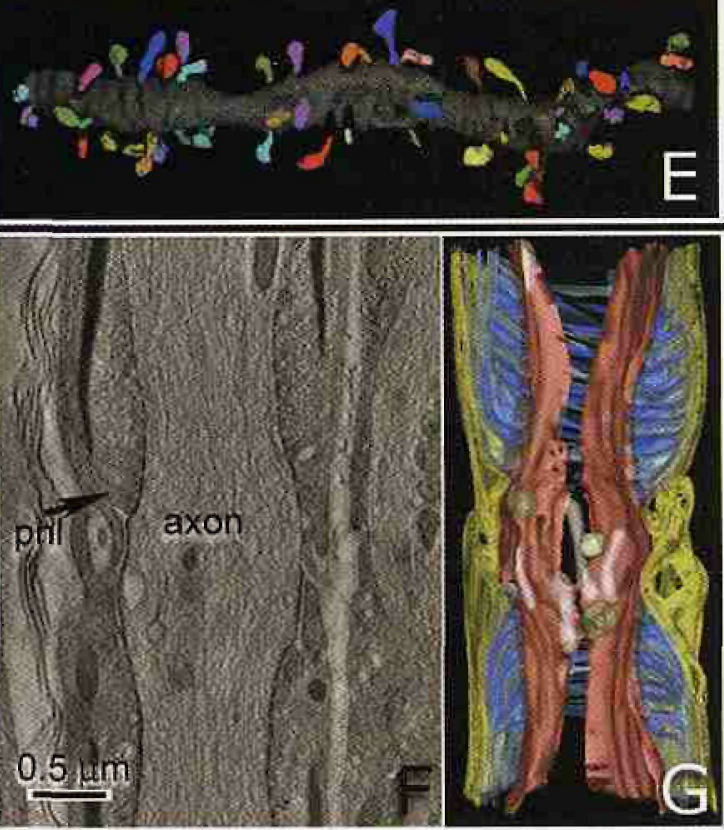

(A)

and (B) are from hippocampal neurons ion the brain and have been selectively stained for actin with an dic spines as shown in green in the confocal micrograph in (A). The red label is an injected es (as indicated by the arrow) but neighboring structures such as synaptic vesicles in (B). Panels Contain one image from the tilt series (C), a volume rendering of the $3 D$ reconstruction (D) and a the brain (F). The images shown in (F) and (G) are from a volume containing a node of Ranvier in dorsal roots of the peripheral nenvous system. Panel $(\mathrm{F})$ is a slice from the reconstruction demonstrating great complexity and details while panel (G) is a visualization of many of the membrane compartments that have been traced, segmented and surface rendered so as to help in interpretation of this complex unit consisting of the axon and two Schwann cells ( $p n l=$ paranodal loops).

microscopic live imaging techniques continues to reveal a level of cellular dynamism that belies the static view of the nervous system we typically see in electron micrographs. Key questions in neurobiology, e.g., the role of receptor trafficking in synaptic plasticity, require the ability to follow dynamic processes in the light microscope followed by additional analysis at the ultrastructural level

NCMIR has pioneered the use of fluorescence photooxidation as a staining technique for correlated light and electron microscopic imaging $(4 ; 29-31)$. Reactive oxygen, generated when fluorescent compounds are strongly excited, drives the oxidation of diaminobenzidine (DAB) into an insoluble polymer that can be rendered electron-dense by treatment with osmium tetroxide $(32 ; 33)$. We made fluorescence photooxidation compatible with immunolabeling, small molecule specific labeling and in situ hybridization ${ }_{1}$ using the fluorophore eosin, a brominated derivative of fluorescein $(29 ; 34)$. This method has the advantage of correlating low resolution analysis at the light microscope to the finer ultrastructural details obtainable at the electron microscope. The specificity of 

the photooxidation-generated labeling and localization is outstanding compared to enzyme-based methods due to the limited spread of the reaction product. The reactive oxygen specles are relatively short-lived and do not diffuse far from the site of production while at the same time, the extensive cross-linking of tissue minimizes the spread of the reaction product.

Critical improvements to the tomographic process are occurring at every step from specimen to analysis. These include: further development of methods for increasing contrast in specimens, automation of data collection, creating mosaics for larger fields as well as development of larger CCD cameras (>16 mega pixels), energy filtering improvements in image alignment and refinement of volumes, faster volume computation using parallel processing, corrections for large contrast transfer function (CTF) values especially at high tilts, improved auto-segmentation algorithms and applications of noise and smoothing filters for improved interpretation and segmentation of tomograms. In addition, many researchers are moving forward with using their tomographic reconstructions as starting models for cellular function simulations $(35 ; 36)$ and also depositing their structures into databases to be used as data inputs for physiological modeling, algorithm development and further structural analyses (37). In conclusion, while the technique has gained recognition in such high profile joumals as Science and Nature, we have not yet seen the pinnacle of electron tomography and we expect this imaging technology to yleld significant and far-reaching discoveries for years to come (38).

\section{References and Notes:}

1. McEwen BF, Marko M (2001) The emergence of electron tomography as an important tool for investigating cellular ultrastructure. $J$ Histochem Cytochem 49:553-564.

2. Baumeister W, Grimm R, Walz J (1999) Electron tomography of molecules and cells. Trend's in Cell Biology 9:81-85.

3. Steven AC, Aebi U (2003) The next ice age: cryo-electron tomography of intact cells. Trends Ce/l Biol 13:107-110.

4. Martone ME, Deerinck TJ, Young SJ, Ellisman ME (1999) Three-dimensional protein localization using high voltage electron microscopy. Acta Histochemica Cytochemica 32:35-43.

5. Bohm J, Frangakis AS, Hegerl R, Nickell S, Typke D, Baumeister W (2000) Toward detecting and identifying macromolecules in a cellular context: template matching applied to electron tomograms. PNAS 97: $14245-14250$.

6. Medalia O, Weber I, Frangakis AS, Nicastro D, Gerisch G, Baumeiste W (2002) Macromolecular architecture in eukaryotic cells visualized by cryoelectron tomography. Science 298:1209-1213.

7. Harlow ML, Ress D, Stoschek A, Marshall RM, McMahan UJ (2001) The architecture of active zone material at the frog's neuromuscular junction. Nature 409:479-484

8. Science (2002), 298:2297-303

9. The contact URL's for the three Research Resources are: http: //bio3d.colorado.edu, http://www.wadsworth.org/rvbc and 10 Frank J, ed (1992) Electron Tomography. New York: Plenum.

11. Radernacher M (1992) Weighted back-projection methods. In: Electron Tomography (Frank J, ed), pp 91-115. New York: Plenum Press.

12. Ladinsky MS, Kremer JR, Furcinitti RS, McIntosh JR, Howell KE (1994) HVEM tomography of the trans-Golgi network: structural insights and identification of a lace-like vesicle coat. JCB 127:29-38.

13. Soto GE, Young SJ, Martone ME, Deerinck TJ, Lamont S, Carragher BO, Hama K, Ellisman MH (1994) Serial section electron tomography a method for three-dimensional reconstruction of large structures. Neuroimage 1:230-243.

14. Marsh BJ, Mastronarde DN, Buttle KF, Howell KE, Mclntosh JR (2001) Organellar relationships in the Golgi region of the pancreatic beta cell line, HIT-T15, visualized by high resolution electron tomography. Proc Natl Acad Sci U S A 98:2399-2406.

15. Martone ME, Deerinck TJ, Yamada N, Bushong E, Ellisman MH (2000) Correlated 3D light and electron microscopy: Use of high voltage electron microscopy and electron tomography for imaging large biological structures. Journal of Histotechnology 23:261-270.

16. Wilson CJ, Mastronarde DN, McEwen B, Frank J (1992) Measurement of neuronal surface area using high-voltage electron microscope tomography. Neuroimage $1: 11-22$.
17. Mastronarde DN (1997) Dual-axis tomography: an approach with alignment methods that preserve resolution. J Struct Biol 120:343-352.

18. Penczek P, Marko M, Buttle K, Frank J (1995) Double-tilt electron tomography. Ultramicroscopy 60:393-410.

19. The Shannon sampling criterion dictates that the minimum theoretical resolution attainable is twice that of the pixel sampling size. In practice, the pixel sampling of digitized images or digital CCD images must be over-sampled (e.g. at least 3-4 times the desired resolution).

20. McEwen BF; Downing KH, Glaeser RM (1995) The relevance of dosefractionation in tomography of radiation-sensitive specimens. Ultramicroscopy 60:357-373.

21. Saxton WO, Baumeister W. Hahn M (1984) Three-dimensional reconstruction of imperfect two-dimensional crystals. Ultramicroscopy 13: 57-70.

22. Kremer JR, Mastronarde DN, McIntosh JR (1996) Computer visualization of three-dimensional image data using IMOD. J Struct Biol 116:71-76.

23. Fung JC, Llu W, de Ruijter WJ, Chen H, Abbey CK, Sedat JW, Agard DA (1996) Toward fully automated high-resolution electron tomography. $\checkmark$ Struct Biol 116:181-189.

24. Peltier ST, Lin AW, Lee D, Mock S, Lamont S, Molina T, Wong M, Dai L, Martone ME, Ellisman MH (2002) The telescience portal for advanced tomography applications. Journal of Parallel and Distributed Computing: Computational Grids in press.

25. Nicastro D, Frangakis AS, Typke D, Baumeister W (2000) Cryo-electron tomography of neurospora mitochondria. J Struct Biol 129:48-56.

26. Frank J, Wagenknecht T, McEwen BF, Marko M, Hsieh CE, Mannella $\mathrm{CA}$ (2002) Three-dimensional imaging of biological complexity. J Struct Biol 138:85-91.

27. Grimm R, Barmann M, Hackl W, Typke D, Sackmann E, Baumeister W (1997) Energy filtered electron tomography of ice-embedded actin and vesicles. Biophys J 72:482-489.

28. Hsieh CE, Marko M, Frank J, Mannella CA (2002) Electron tomographic analysis of frozen-hydrated tissue sections. J Struct Blol 138:63-73.

29. Deerinck TJ, Martone ME, Lev-Ram V, Green DPL, Tsien RY, Spector DL, Huang S, Ellisman MH (1994) Fluorescence photooxidation with eosin: a method for high resolution immunolocalization and in situ hybidization detect for light and electron microscopy. JCB 126:910-910.

30. Huang S, Deerinck TJ, Ellisman MH, Spector DL (1997) The dynamic organization of the perinucleolar compartment in the cell nucleus. JCB 137:965-974.

31. Capani F, Ellisman MH, Martone ME (2001) Filamentous actin is concentrated in specific subpopulations of neuronal and glial structures in rat central nervous system. Brain Res 923:1-11.

32. Maranto A (1982) Neuronal mapping: a photooxidation reaction makes Lucifer Yellow useful for electron microscopy. Science 217:953-955.

33. Sandell JH, Masland RH (1988) Photoconversion ofsome fluorescent markers to a diaminobenzidine product. JHistCyto $36: 555-559$.

34. Capani F, Deerinck TJ, Ellisman MH, Bushong E, Bobik M, Martone ME (2001) Phalloidin-eosin followed by photo-oxidation: a novel method for localizing F-actin at the light and electron microscopic levels. J Histochem Cytochem 49:1351-1361.

35. Stiles JR, Bartol TM, Salpeter MM, Salpeter EE, Sejnowski TJ (2001) Synaptic variability: new insights from reconstructions and Monte Carlo simulations with MCell. In: Synapses (Cowan WM, Sudhof TC, Stevens CF, eds): Johns Hopkins University Press.

36. Loew LM, Schaff JC (2001) The Virtual Cell: a software environment for computational cell biology. Trends Biotechnol 19:401-406.

37. c.f. the Cell Centered Database http://ncmir.ucsd.edu/CCDB and the Macromolecular Structure Database http://www.ebi.ac.uk'

38. In addition to the references cited above, there are several review and research articles that are excellent resources for the field of electron tomography. These include the Journal of Structural Biology volumes 120 and 138; Baumeister \& Steven (2000), Trends Biochem Scl, 25: 624-31; Baumeister, Grimm, \& Walz, Trends Cell Biol, (1999) 9: 81-85; Martone et al., (1999) Acta Histochem. Cytochem 32: 35-43: McEwen \& Frank. (2001) Curr Opin Neurobiol. 11: 594-600; and McEwen \& Marko, J. Histochem. Cytochem. (2001) 49:553-64; The preceding article was adapted from a Microscopy Society of America 2002 annual meeting abstract by Sosinsky and the material was presented as a biological tutorial 


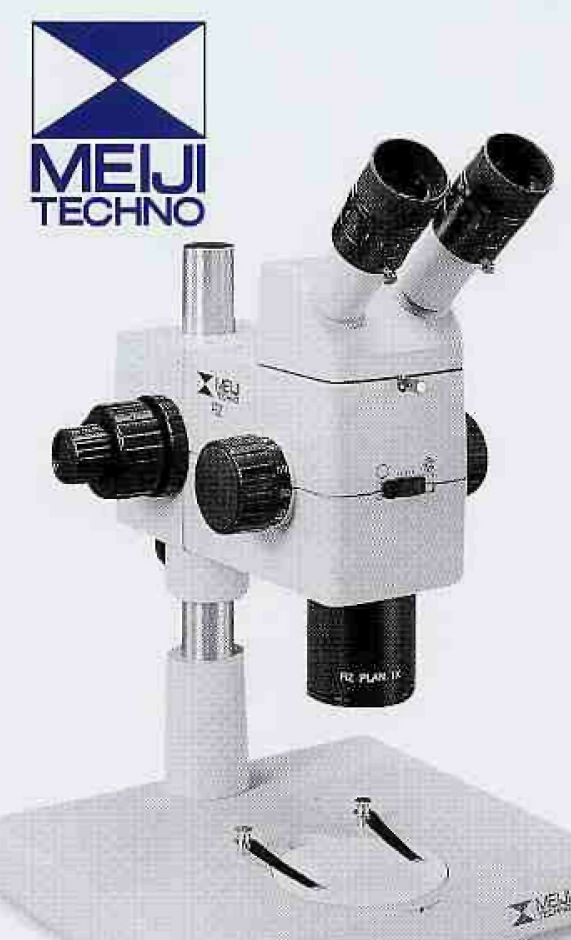

\section{The Meiji RZ Series of Research Stereo Microscopes.}

If you are looking for precision, durability, quality and value in a high performance Stereo Microscope, we invite you to take a closer look at Meiji's RZ Series of Research Stereo Microscopes.

The RZ Series modular system design allows you the freedom to create an ideal instrument for your specific need or application. Featuring a 10:1 zoom ratio, variable double iris diaphragm, and positive detente click stops at 12 positions of magnification. A full range of optional accessories is available, including: Video and photo-micrographic systems, brightfield-darkfield transmitted light stands, ergonomic binocular head, drawing attachment, multiple interchangeable objectives and wide-field eyepieces. Complete system versatility backed by a "Limited Lifetime Warranty."

For more information on these economically priced Stereo Microscopes, please call, FAX, write us or log on to our website today.

\section{MEIJI TECHNO AMERICA}

2186 Bering Drive, San Jose, CA 95131 ,

Tel: 408.428 .9654 , FAX: 408.428 .0472

Toll Free Telephone: 800.832 .0060 or visit our website at www.meijitechno.com

\section{STOP HASSLING WTH \\ MULTIPLE SERVIGE CONTRAGTS!}

START by putting all of your instruments under one service contract with MAS (regardless of make or model). Our expert EM SERVICE GROUP has the knowledge and skills to keep your instrument working at its best.

\section{TEM'S/SEMS PREP EQUIPMENT SPECIAL SERVIGES}

HITACHI TOPCON JEOL ISI

AMRAY CAMBRIDGE

\section{VACUUM COATERS}

PLASMA ASHERS

SPUTTER COATERS

ION MILLS
STAGES

BEAM BLANKERS

CUSTOM DEVICES

\section{$N E W \quad N=W \quad N E W$}

\section{Authorized service representatives} for Gatan preparation equipment and Topcon TEM'S.
Contracts and On-Demand

Emergency Service at

Reasonable Rates from

Factory Trained Specialists.

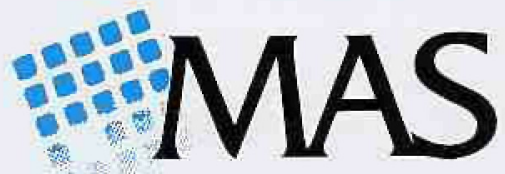

3945 Lakefield Court Suwanec, Georgii $30024 \quad 770-866-3200$ FAX 770-866-3259 616 Hutton Street Suite 101 Raleigh, North Cirolina 27606 919-829-7041 FAX 919829-5518 ADVANCED ANALYTICAL PRODUCTS AND SERVICES 